\title{
Evidence of Rapid Gender Processing Revealed by ERSP
}

\author{
Zhong-Lei Gu, Li-Chen Shi and Bao-Liang Lu* Senior Member, IEEE
}

\begin{abstract}
In this research, we used EEG signals to analyze gender processing with the ERSP method. Not only facial images, but also images of clothing and shoes, were used. We applied the ICA method to obtain a gender-related component which appeared quite significant in the majority of electrode sites for the occipital lobe. This showed differences of energy between the two genders, even for the clothing and shoe images. Our results indicate that not only facial gender processing, but also a gender discrimination task for objects influences the energy of EEGs from $50 \mathrm{~ms}$ after the onset of stimuli at all frequencies, especially lower band. This provides convincing evidence for rapidity of gender processing.
\end{abstract}

\section{INTRODUCTION}

Gender discrimination is involved in many parts of life, such as socializing, viewing photographs and picking clothes; it is obviously important.

Cases of prosopagnosia show that when the brain is damaged a patient, although not able to recognize faces, is capable of discriminating a person's gender and race by looking at his or her face with few exceptions [12][5][3]. In 1986, thanks to series of behavioral experiments, daily observation and clinical outcome, Bruce and Young [2] supposes that there are two separate channels to process human faces in parallel: one to recognize people and the other to deal with facial age, race, gender and emotions. Face-specific scalp ERP components on the posterior temporal lobe peaking at $\approx 170 \mathrm{~ms}$ from stimulus onset (N170) are associated with the mechanisms of structural analysis of facial features [1] prior to identification.

On the other hand, the methods proposed in pattern recognition usually follow a standard two-phase process, namely feature extraction phase and pattern classification phase. There are no obvious differences between these methods for face recognition and gender classification. However, according to the behaviors of prosopagnosia and the processing time of humans, these two processing procedures show a great difference.

Hence it is of great significance to study the physiological mechanism and process of gender discrimination of the human brain and imbed this mechanism into practical applications. Moreover, the understanding of this mechanism will lead to progress in the understanding of the human vision system.

This work was partially supported by the National Natural Science Foundation of China (Grant No. 90820018), the National Basic Research Program of China (Grant No. 2009CB320901), the Science and Technology Commission of Shanghai Municipality (Grant No. 09511502400), and the European Union Seventh Framework Programme (Grant No. 247619).

Z. L. Gu, L. C. Shi and B. L. Lu are with the Department of Computer Science and Engineering, Shanghai Jiao Tong University, Shanghai, 200240, China, and MOE-Microsoft Key Laboratory for Intelligent Computing and Intelligent Systems, Shanghai Jiao Tong University, Shanghai, 200240, China. *Corresponding author: bllues jtu.edu. cn
In this paper, we provide several pieces of evidence which indicate gender processing takes place prior to N170, by observing significant differences among induced ERSP from EEGs recorded while showing several gender discriminable images to subjects.

\section{RELATED WORK}

Research results in neurophysiology show that human brains have several functional and anatomical differences while identifying human faces and other objects. In the temporal cortex of macaque brains, especially in the inferior temporal gyrus and the superior temporal sulcus, the cells which are related to face identification have been discovered [14]. This mass of cells produces a strong reaction when given a stimulus of a human face. Based on the fMRI records of patients, Kanwisher [6] points out that the fusiform gyrus area is activated when given a stimulus of a human face, and this area has no reaction when the stimuli are buildings, mixed faces and hands. In research of identification for eastern and western faces [15], there is evidence that western faces have fewer distinguishing features than eastern faces. This point provides an electronic physiology explanation for the confusion of different racial face identification. It also shows that the racial characteristics do not include the external characteristics of faces in the mixed effect theory. Yonlande et al. [10] recently showed that, N170 (in the ERP experiment, the negative peak appearing $170 \mathrm{~ms}$ after the stimulus ) has nothing to do with the processing of gender information. He also showed that, the wave in 45-85 $\mathrm{ms}$ of the judgment for hands and faces has an obvious difference, and in 145-185 ms of the judgment for the faces also has a difference. Other research on the gender information of faces has discovered that, as early as in $40 \mathrm{~ms}$ or $60 \mathrm{~ms}$, although the electronic activities for gender processing do not have an obvious reaction, they can be regarded as a rough classification for the rapid processing in early time [9]. Also, the change of the electrical activity in the occipital lobe and the frontal lobe $140 \mathrm{~ms}$ to $260 \mathrm{~ms}$ after the stimulation shows that in this stage the cortex in these areas are very active in the processing of the gender information contained in the faces.

\section{MATERIALS AND SUBJECTS}

\section{A. Participants}

Twenty-three normal, right-handed participants (mean age $24 \pm 1$ years, twelve males and eleven females) with normal or corrected-to-normal vision participated as paid volunteers. All were fully informed of the recording technique, which was noninvasive. 


\section{B. Stimuli}

Nine hundred photographs were digitally stored. They included four stimulus types (see Fig. 1): human faces with no eyeglasses, human faces with eyeglasses, pictures of shoes and pictures of clothing. And faces are of three different subtypes, university students' faces (mean age $18 \pm 1$ years, ID Card), company employees' faces (mean age $27 \pm 4$ years, ID Card) and less formal pictures of faces from the Internet. The face photos were all above chest and presented in a roughly front view. Across categories, the stimuli were matched for luminance and were of equal size $(430 \times 650$ pixel). The stimuli were presented foveally, subtending a visual angle of $6.7^{\circ}$, for $300 \mathrm{~ms}$ at a rate of one every 1.4$1.7 \mathrm{~s}$ randomly. A fixation point was present at the center of the screen between each presentation. There was a fifteen second break for every 50 stimuli. No stimulus was repeated and were unknown to the subjects. The sequence of stimuli for each subject was the same for ensuring each subject experiencing the same.

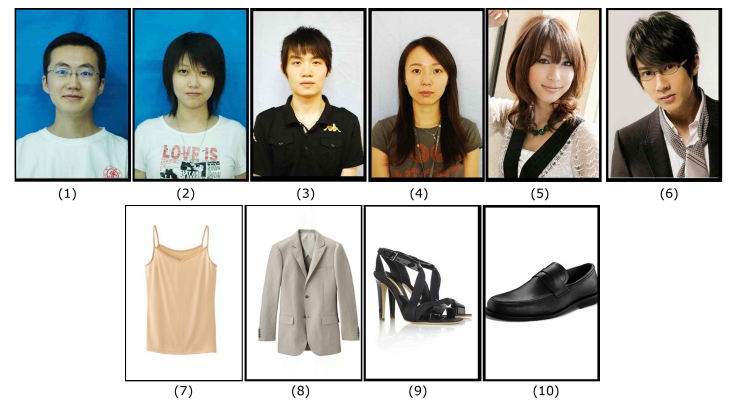

Fig.1. Excerpt of a sequence of stimuli. (1), (2): university students. (3), (4): company employee. (5), (6): faces from Internet. (7), (8): clothing. (9), (10): shoes

We split these nine sessions into several groups.

1) University students' faces: In sessions 1 and 2, the subjects were asked to complete a discrimination task not involving gender. Target and non-target stimuli were of only one gender, and the task was to tell whether the person was wearing glasses or not. We suggest that gender discrimination did not explicitly take place in these two sessions. All the stimuli of these two sessions were ID card images of freshman university students.

2) Company employees' faces: In sessions 4 and 5, the subjects had to discriminate by gender. The targets were either male (session 4) or female (session 5) faces to be distinguished from those of the opposite sex. The pictures in both sessions were ID card images of company employees.

3) Faces from the Internet: In sessions 7 and 8, the subjects also needed to recognize the gender, but with abundant background and peripheral information.

4) Shoes and clothing: In session 6, the subjects are required to judge whether clothing or shoes are presented on the screen. And in session 9, they need to tell women's clothing from men's. In the former session, subjects judge gender incidentally, but in the second it is central.

\section{Procedure}

The experiment consisted of nine consecutive sessions, containing 100 stimuli each. In all the sessions, subjects
TABLE I

EXPERIMENTAL PARADIGM

\begin{tabular}{lll}
\hline No. & Non-target stimuli (n) & Target stimuli (n) \\
\hline 1 & Male with glasses (80) & Male without glasses (20) \\
2 & Female without glasses (80) & Female with glasses (20) \\
3 & Male faces (50) & Female faces (50) \\
4 & Male faces (80) & Female faces (20) \\
5 & Female faces (80) & Male faces (20) \\
6 & Clothing (40 Male + 40 Female) & Shoes (10 Male + 10 Female) \\
7 & Casual female faces (80) & Casual male faces (20) \\
8 & Casual male faces (80) & Casual female faces (20) \\
9 & Female clothing (80) & Male clothing (20) \\
\hline
\end{tabular}

performed an oddball detection task reporting by pressing a button. Target items ( $20 \%$ of total) were delivered randomly among non-target items (see Table I for details). Due to expected tiring status for subject, the balancing parts for session 6 and 9 (with the following percentage of nontarget/target stimuli: shoes (40 male +40 female), clothing (20 male +20 female); male clothing(80), female clothing (20)), were missing. This will be verified in the further study.

\section{EEG recording}

Subjects were fitted with a 64-channel electrode cap during the experiment. The $\mathrm{Ag} / \mathrm{AgCl}$ electrodes were mounted inside the cap with reference on top of the scalp. The electrodes were arranged according to the international 10-20 system. The contact impedance between electrodes and skin was kept to a value less than $5 k \Omega$. The EEG data were recorded with 32-bit quantization level at a sampling rate of $1000 \mathrm{~Hz}$ by the NeuroScan Scan software.

\section{METHOD}

\section{A. Artifact Detection}

The time wave and energy of each trial (the segment of EEG from $-100 \mathrm{~ms}$ to $499 \mathrm{~ms}$ after the onset of the stimuli) were visually checked. Trials seriously contaminated by electromyogram (EMG) or electrooculogram (EOG) were removed manually. Bad or anomalous channels were recorded and removed during the experiment and data analysis. Those trails or channels typically showed larger amplitude waves and higher energy compared to normal ones.

\section{B. Event-related Spectral Perturbation}

Event-related Spectral Perturbation (ERSP) is a method of studying the EEG spectrum induced by the onset of stimuli. It reveals aspects of event-related brain dynamics not contained in the ERP average of the same response epoch [13]. We applied ERSP both to all trials in a session and to all trials for each gender with one subject.

Inter-trail coherence (ITC) is computed together with ERSP at all frequencies. A significant ITC based on the EEG activity at a given time and frequency indicates an algorithm that is phase-locked; all trials give similar result.

\section{Independent Component Analysis}

Independent component analysis (ICA) is a special case of blind source separation; it is suitable for and widely used in EEG decomposition. ICA reconstructs the mixed signal by maximizing the statistical independence of the estimated components. More specifically, where $x_{i}$ are the observed 
components and $s_{i}$ the independent sources, where $i=1 . . n$, and $\mathrm{n}$ is the number of electrodes, call the mixing weight matrix A. Then for each $x_{i}$, we have $x_{i}=a_{i, 1} s_{1}+\ldots+$ $a_{i, k} s_{k}+\ldots+a_{i, n} s_{n}$. Now, ICA finds the optimal solution of $\mathrm{A}$, under the premise of ensuring minimization of mutual information or maximization of non-Gaussianity.

\section{RESULTS}

We apply ERSP to both the original EEG and the ICA components. The result of the former appears obviously different between male and female stimuli, and for the latter, we found two components from different kinds of stimuli have the same topographic mapping.

\section{A. ERSP: facial gender}

The results of ERSP (bootstrapping significance $<0.01$ ) on the original EEG and the corresponding ITC for facial gender are shown in Fig. 2. One can observe two interesting facts from the figure. First, At around 100ms after onset of stimuli, the energy induced by female stimuli is significantly larger than for male stimuli in almost all frequency bands. Another significant energy gap between two stimuli is at the beta band $(13-24 \mathrm{~Hz})$, around $150 \mathrm{~ms}$. Secondly, the summits of ITC can be observed around $100 \mathrm{~ms}$ and $170 \mathrm{~ms}$ for both sessions, which indicates high correlation among trials. And correlation among trails is also high around $50 \mathrm{~ms}$ in male session, but not in female one. A high correlation among trials appears after $200 \mathrm{~ms}$ for female sessions, but seldom for males. Considering these phenomena occurred in both no-gender and intentional tasks, we can conclude that the statistically significant differences in time and frequency are relevant to facial gender processing.

\section{B. ERSP: clothing and shoes}

The results of ERSP (bootstrapping significance $<0.01$ ) on the original EEG and the corresponding ITC for clothing and shoes are shown in Fig. 3. Compared with the conclusion of facial gender, the differences between ERSP of the two kinds of stimuli almost fade away. However, there is great similarity between their results for ITC. Hence the result indicates that the significant time and frequency differences are not only relevant to the facial specific P100 and N170, but also related to gender processing.

\section{ERSP: pooling the same gender}

We put some stimuli from a single subject together, including session $1,2,4,5,7,8$, which contain 240 male and 240 female stimuli. The aim is to find the general character for face gender processing by the method of averaging EEGs from different kinds of stimuli but same gender. The results are shown in Fig. 4. One can clearly note the significant difference around $100 \mathrm{~ms}$ for each subject but at different a frequency band.

\section{Statistics across subjects}

We propose a simple statistic to figure out the most notable electrode sites (exclude frontal electrodes) across the subjects. In detail, for an electrode, we calculate the frequency of significant difference of ERSP between two genders among all subjects. The results are shown in Fig.5.
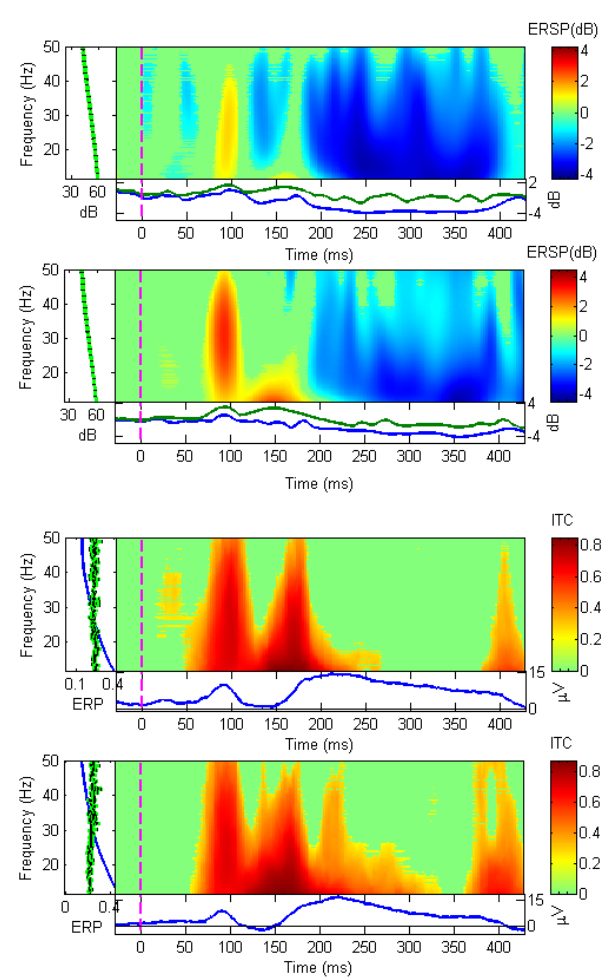

Fig.2. Results of ERSP and ITC from a single subject (female) for facial gender at electrode P4. (a) ERSP result: the above is of session 1 (male stimuli) and the bottom is of session 2 (female stimuli). The lower part of each panel indicates the ERSP envelope (low and high mean $\mathrm{dB}$ values, relative to baseline, at each time in the epoch [4]). (b): ITC result: the above is of session 1 (male stimuli) and the bottom is of session 2 (female stimuli).
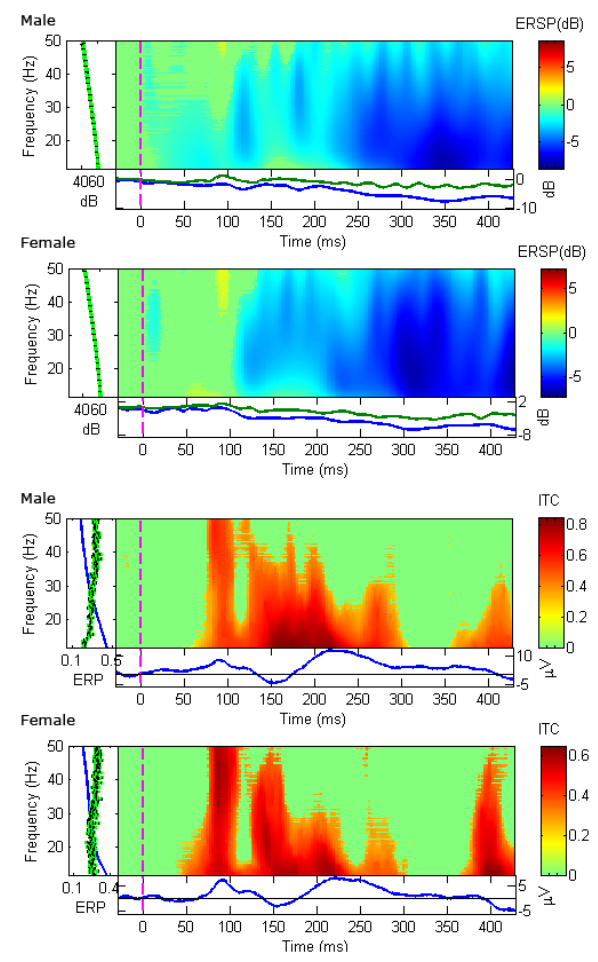

Fig.3. Results of ERSP and ITC from a single subject (female) for clothing and shoes at electrode P4. (a): ERSP result: the above is of men's clothing and shoes and the bottom is of women's clothing and shoes. (b): ITC result: the above is of men's clothing and shoes and the bottom is of women's clothing and shoes. 

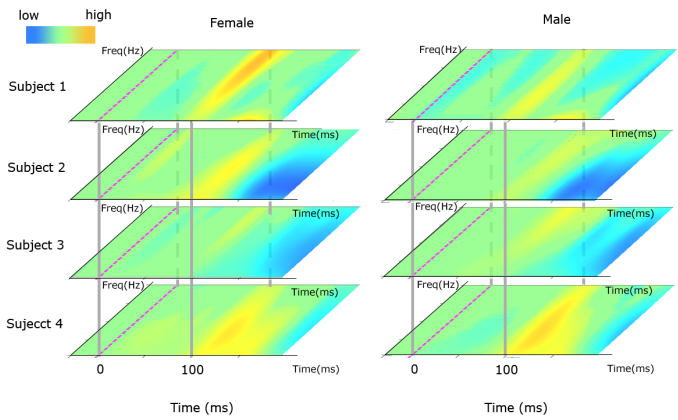

Fig.4. ERSP results across the sessions at electrode P4. Each layer represents data for one subject. The left part shows the ERSP induced by female stimuli, and the right part shows the ERSP induced by male stimuli.

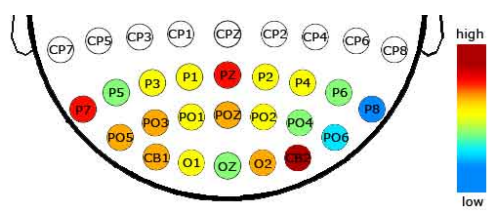

Fig.5. The results indicate notable electrodes across the subjects. The color bar denotes the frequency of significant difference.

\section{E. ERSP: ICA Components}

Inspired by observations of large energy appearing at the occipital lobe from the stimuli onset, we propose an ICA method to decompose the data to find the key sources of the task. The results are shown in Figs. 6 and 7.

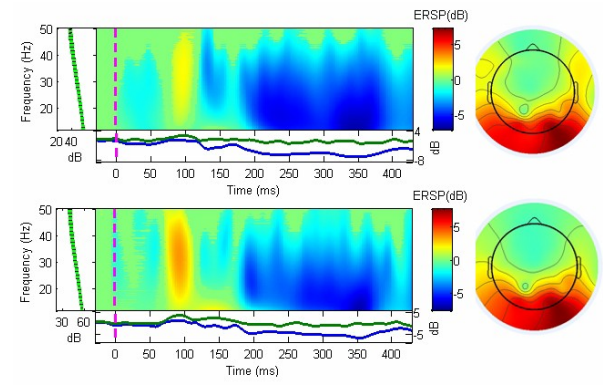

Fig.6. Results of ICA components' ERSP. The above is the component from session 1 and the bottom is the component from session 2 . The right parts are the corresponding topographic mappings of the components.

\section{CONCLUSION}

\section{A. Gender Processing}

Note that significant differences between both genders can be observed by ERSP. Moreover, for each subject, with the method of ICA a high correlation among trails 50ms after onset of stimuli appears more clearly. These results partly reveal the evidence for rapidity of gender processing in humans, especially in the lower bands, considered related to cognitive activities.

As the observation of Fig. 5, the electrode sites found by our experiment, $\mathrm{P} 7$ and $\mathrm{CB} 2$, are consistent with previous results [10] [7], while PZ has not been reported before. Further evidence for this phenomenon is needed.

\section{B. Clothing and Shoes}

In session 5, subjects were involved in tasks not involving gender, discriminating between clothes and shoes, while in

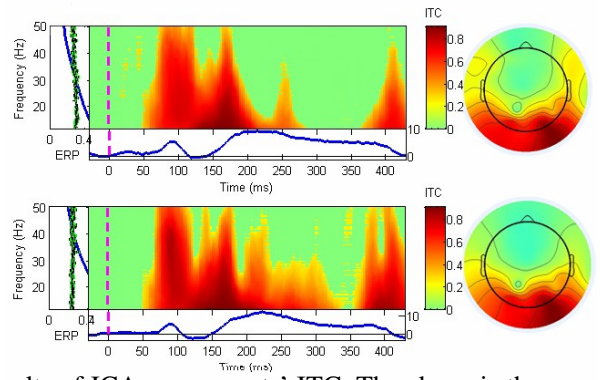

Fig.7. Results of ICA components' ITC. The above is the component from session 1 and the bottom is the component from session 2. The right parts are the corresponding topographic mappings of the components.

session 9, subjects intentionally judge whether the clothes are for female or not. Both of these results show that, compared to the results about human faces, the relation among trails around $100 \mathrm{~ms}$ and $170 \mathrm{~ms}$ is just slightly weaker. These two time points are usually regarded as facial specific ERP, P100 and N170. So these two time points are related to the gender processing, not only for the human faces.

\section{REFERENCES}

[1] S. Bentin, T. Allison, A. Puce, A. Perez, G. McCarthy, Electrophysiological studies of face perception in humans, Congnit. Neurosci., 1996, 8, 551-565,

[2] V. Bruce and A. Young, Understanding face recognition. Psychol., 1986, 77, 305327.

[3] G.A. Carlesimo and C. Caltagirone, Components in the visual processing of known and unknown faces. Clin. Exp. Neuropsychol., 1995, 17, 691705.

[4] A. Delorme and S. Makeig, EEGLAB: an open source toolbox for analysis of single-trial EEG dynamics. Journal of Neuroscience Methods, 2004, 134:9-21.

[5] E. De Renzi, M.G. Bonacini, P. Faglioni, Right posterior braindamaged patients are poor at assessing the age of a face. Neuropsychologia, 1989, 27, 839-848.

[6] N. Kanwisher, J. McDermott, M. Chun, The fusiform face area: a module in human extrastriate cortex specialized for face perception. Journal of Neuroscience, 1997, 17(11):4302

[7] C. Kaul, G. Rees, A. Ishai, The gender of face stimuli is represented in multiple regions in the human brain. Frontiers in Human Neuroscience[J], 2011, 10(3389)

[8] F. Kranz and A. Ishai, Face perception is modulated by sexual preference. Curr. Biol., vol. 16, 2006, pp 63-68.

[9] Y.F. Luo, S. Zhou, Y. Jia, T.M. Lu. Facial sexual feature processing revealed by spatiotempraral pattern of event-related potentials. Chinese Journal of Neuromedicine, 2008, 7(7)

[10] Y. Mouchetant-Rostaing, M. Giard, S. Bentin, et al, Neurophysiological correlates of face gender processing in humans. European Journal of Neuroscience, 2000, 12(1):303-310

[11] Y. Mouchetant-Rostaing and M. Giard, Electrophysiological correlates of age and gender perception and human faces. Journal of Cognitive Neuroscience, 2003, 15(6):900-910

[12] F. Newcombe and W.R. Russel, Dissociated visual perceptual and spatial deficits in focal lesions on the right hemisphere. Neurol. Neurosurg. Psychiatr., 1969, 32, 73-81.

[13] S. Makeig, Auditory event-related dynamics of the EEG spectrum and effects of exposure to tones. Electroencephalogy and Clinical Neurophysiology, 86(4), 1993, PP 283-293.

[14] Perrett D, Rolls E, Cann W, Visual neurones responsive to faces in the monkey temporal cortex. Experimental Brain Research, 1982, 47(3):329-342.

[15] X.H. Peng, Y.J. Luo, J.H. Wei, G.F. Wang, Recognition of Eastern and Western Faces with Internal and External Features: An ERP Study. Space Medicine and Medical Engineering, 2003, 16(2). 